\title{
Pertinence des prévisions météorologiques à l'échelle kilométrique pour la modélisation du manteau neigeux en montagne
}

\author{
Ingrid Dombrowski-Etchevers' ${ }^{1}$, Vincent Vionnet ${ }^{2, *}$, Louis Quéno $^{2}$ \\ 1 Centre national de recherches météorologiques, Météo-France / CNRS, \\ Toulouse \\ 2 Centre d'études de la neige, Centre national de recherches météorologiques, \\ Météo-France / CNRS, Grenoble \\ * Maintenant à : Centre for Hydrology, University of Saskatchewan, \\ Saskatoon, Canada \\ ingrid.etchevers@meteo.fr
}

\section{Résumé}

L'augmentation de la résolution horizontale des modèles météorologiques offre des perspectives pour la modélisation du manteau neigeux en zone de montagne. La capacité du modèle météorologique kilométrique Arome à reproduire les conditions météorologiques et leur grande variabilité a été étudiée sur les Alpes françaises et les Pyrénées, sur les quatre hivers 2010/2011 à 2013/2014. Les résultats ont montré un biais froid d'Arome en altitude pour les températures. L'impact du relief sur les précipitations est bien représenté par Arome, même si les quantités ont tendance à être surestimées par rapport aux observations. Des simulations du manteau neigeux par Surfex-Crocus alimenté par les prévisions Arome ont ensuite été réalisées sur les Alpes françaises et les Pyrénées. Arome/Surfex-Crocus surestime les hauteurs de neige, le transport de neige par le vent n'étant pas représenté et la fonte nivale sousestimée. En revanche, la répartition spatiale de l'enneigement simulé est en bonne adéquation avec la fraction neigeuse issue de TerraModis. Combinée à une analyse météorologique adaptée au relief, Arome/Surfex-Crocus pourrait fournir une modélisation pertinente du manteau neigeux.
《 En montagne, le temps (sousentendu la météorologie) peut changer rapidement et être très différent d'un versant à l'autre » (figure 1). Cet adage est souvent rappelé par les pratiquants, professionnels ou non, d'activités en montagne. En effet, le relief complexe, la topographie locale, l'altitude, la pente des versants ou encore leur orientation par rapport au flux atmosphérique influencent les conditions météorologiques rencontrées en zone de montagne (e.g. Martin et al., 1994 ; Chow et al., 2013). La prise en compte de cette variabilité météorologique, à la fois temporelle et spatiale, est un enjeu

majeur pour la prévision des conditions nivologiques et hydrologiques en montagne.

Les conditions atmosphériques telles que la température, la phase des précipitations, le vent, les rayonnements solaire et infrarouge incidents, fortement influencées par les conditions de surface et l'altitude déterminent la variabilité spatiale du manteau neigeux. À petite échelle (moins de $100 \mathrm{~m}$ ), des processus comme le transport de neige par le vent ou les avalanches jouent un rôle décisif sur la répartition spatiale du couvert neigeux. À l'échelle du versant,

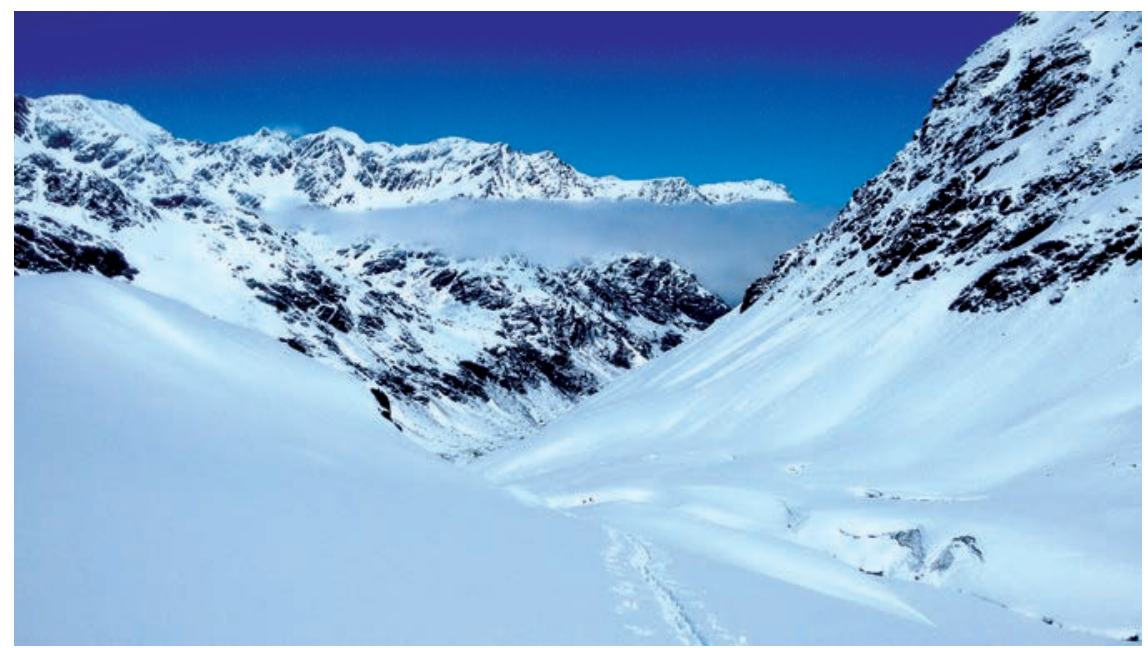

Figure 1. Mer de nuages vers 2000 mètres d'altitude. Montée vers le col d'Arinsal, vallée du Vicdessos, Ariège, Pyrénées, avril 2016. Crédit photo : P. Etchevers. 


\section{Abstract}

\section{Pertinence of kilometric-scale weather forecasts for snowpack modeling in mountains}

\begin{abstract}
The increasing of the horizontal meteorological models resolution offers prospects for the snowpack modeling in mountain areas. The ability of the meteorological kilometric-scale model Arome to reproduce weather conditions and their great variability was studied in the French Alps and in the Pyrenees over the four winters of 2010-2013. The results showed a cold bias in altitude for the temperatures. The impact of relief on precipitation is well represented by Arome, although quantities tend to be over-estimated relative to measurements. The snowpack simulations by SurfexCrocus driven by Arome forecasts were carried out on the French Alps and the Pyrenees. Arome/SurfexCrocus overestimates snow depths, mainly due to non-simulated windinduced erosion and snowmelt underestimation. However, the spatial distribution of simulated snow cover is in a good agreement whith the snow cover fraction of TerraModis. Associated with a terrainadapted weather analysis, Arome/ Surfex-Crocus could provide relevant modeling of the snowpack.
\end{abstract}

\section{Réanalyses Safran en zone de montagne}

Les réanalyses Safran (Durand et al. 1993, 2009) sont effectuées sur les zones de montagne climatologiquement homogènes dénommées massifs (taille moyenne $930 \mathrm{~km}^{2}$ ), par pas de $300 \mathrm{~m}$ $d^{\prime}$ altitude. Elles utilisent comme ébauche les profils verticaux issus des analyses Arpège à $20 \mathrm{~km}$ et toutes les observations automatiques et manuelles du réseau nivométéorologique disponibles par massif. Les paramètres analysés sont la température et I'humidité relative à $2 \mathrm{~m}$, le vent à $10 \mathrm{~m}$, la nébulosité ainsi que les précipitations (quantité et phase). Le rayonnement solaire (direct et diffus) et le rayonnement infrarouge descendant sont évalués à partir des variables préalablement analysées l'exposition des pentes au rayonnement solaire incident influence fortement l'évolution du manteau neigeux et génère des contrastes marqués de couverture neigeuse entre les versants sud (exposés au soleil) et nord (à l'ombre). Enfin, à plus large échelle, l'influence du relief sur les précipitations (altitude, exposition par rapport aux flux synoptiques) génère des contrastes régionaux marqués en termes d'enneigement. La variabilité du manteau neigeux simulé par un modèle est donc très dépendante de la résolution spatiale des données atmosphériques fournies en entrée.

Aujourd'hui, la prévision des conditions de neige dans les ensembles montagneux français repose sur le système d'analyse et de prévision météorologique Safran (Système d'analyse fournissant des renseignements atmosphériques utiles à la neige ; Durand et al., 1993) qui fournit un forçage atmosphérique à Crocus, modèle de neige implanté dans la plateforme de modélisation de surface Surfex, appelée Surfex-Crocus (Brun et al., 2012 ; Vionnet et al., 2012). Safran produit en analyse et en prévision des champs atmosphériques pour des massifs montagneux climatologiquement homogènes d'environ $900 \mathrm{~km}^{2}$, par pas de 300 m d'altitude (voir encadré 1). Ces champs sont utilisés pour alimenter Surfex- Crocus qui simule l'évolution du manteau neigeux selon ces mêmes résolutions suivant la pente (plat, $20^{\circ}$ et $40^{\circ}$ ) et $1^{\prime}$ exposition (nord, nordest, est, sud-est, sud, sud-ouest, ouest, nord-ouest). Une version étendue de Safran sur la France est également utilisée pour le suivi hydrométéorologique (Soubeyroux et al., 2008).

Cependant, en montagne, cette simulation de l'enneigement par massif et par classe d'altitude, de pente et d'orientation ne permet de représenter que de manière limitée la variabilité spatiale intramassif du manteau neigeux. Afin de raffiner le forçage météorologique fourni à Surfex/Crocus, une alternative à Safran est offerte par le système de prévision numérique du temps (PNT) Arome (Applications de la recherche à l'opérationnel à mésoéchelle ; Bouttier, 2007 ; Seity et al., 2011) opérationnel à Météo-France depuis décembre 2008. Avec sa résolution horizontale kilométrique (2,5 km jusqu'en avril 2015, 1,3 km désormais), Arome permet une représentation améliorée de la topographie par rapport au concept de massif et de bandes d'altitude utilisé par Safran. Arome appartient à la catégorie des systèmes de PNT à haute résolution utilisés pour la prévision du temps à courte échéance dans de nombreux centres météorologiques. De tels systèmes sont utilisés quotidiennement pour fournir des prévisions en zone de montagne. Ainsi, le modèle Cosmo (Baldauf et al., 2011) est utilisé sur les Alpes suisses (résolution kilométrique) et allemandes (résolution 2,8 km). Aux États-Unis, le système HRRR (High Resolution Rapid Refresh, Clark et al., 2012) fournit des prévisions à $3 \mathrm{~km}$ de résolution sur l'ensemble du pays (notamment les Rocheuses). Ces systèmes ont notamment été utilisés lors d'événements à fort enjeu en zone de montagne comme les Jeux olympiques d'hiver de Vancouver en 2010 (Mailhot et al., 2014) et ceux de Sotchi en 2014 (Kiktev et al., 2013).

Le potentiel des systèmes de PNT à haute résolution pour la prévision de l'état du manteau neigeux en zone de montagne est encore à évaluer. C'est l'enjeu de cette étude utilisant les prévisions du modèle Arome pour alimenter le modèle détaillé de manteau neigeux Crocus. Dans un premier temps, les températures et précipitations hivernales prévues par Arome ont été évaluées par rapport aux observations et aux réanalyses Safran, qui sont la meilleure estimation spatialisée des conditions météorologiques en zone montagneuse (Durand et al., 2009) pour quatre hivers ( $\mathrm{du} 1^{\mathrm{er}}$ novembre au 31 mai), dans les Alpes françaises et les Pyrénées. Pour ensuite estimer l'intérêt pour la nivologie et l'hydrologie, les hauteurs de neige et les étendues spatiales du couvert neigeux obtenues par les simulations de Surfex-Crocus forcées par Arome sur la même période et les mêmes massifs ont été comparées aux mesures in situ, satellitaires et à des simulations Surfex-Crocus alimentées par les réanalyses Safran. C'est la synthèse de ces travaux qui est présentée dans cet article. Une analyse détaillée des résultats est par ailleurs disponible dans deux études récentes sur les Alpes françaises (Vionnet et al., 2016) et les Pyrénées (Quéno et al., 2016).

\section{Comportement d'Arome \\ en zone de montagne}

Le modèle météorologique non hydrostatique Arome résulte de la fusion entre les modèles Aladin pour la 
dynamique et l'assimilation (Fischer et al., 2005) et Méso-NH pour la physique (Lafore et al., 1998). Ceci lui permet de mieux prévoir les phénomènes extrêmes comme les orages ou les pluies cévenoles et ceux de fine échelle comme le brouillard ou les brises (maritimes ou de pente) grâce à sa résolution kilométrique. La version évaluée dans cet article est celle à $2,5 \mathrm{~km}$ de résolution horizontale, pour les hivers 2010-2011 à 2013-2014. Dans cette version, Arome utilise une assimilation de données toutes les 3 heures et possède une analyse de surface (température et humidité à $2 \mathrm{~m}$ ) (Brousseau et al., 2008). La couverture neigeuse ainsi que les hauteurs de neige ne sont pas assimilées par le système d'analyse de surface.

Dans cette étude, nous avons évalué les prévisions météorologiques journalières issues du réseau de $0 \mathrm{~h}$ UTC, des échéances $6 \mathrm{~h}$ UTC à $5 \mathrm{~h}$ UTC le lendemain, à la résolution horizontale de $0,025^{\circ}$ (environ 2,5 km). La période d'étude couvre les quatre saisons nivologiques (du $1^{\text {er }}$ novembre au 31 mai) de 2010 à 2014 pour les Alpes françaises et pour les Pyrénées françaises et espagnoles. Au cours de cette période, la base de données utilisée pour générer le relief à 2,5 km utilisé dans Arome a été modifiée. Jusqu'à l'été 2012, le relief dans Arome était issu d'une version à basse résolution $(5 \mathrm{~km})$ de la base de relief GTOPO30. À partir de l'été 2012, GTOPO30 à sa résolution nominale (environ $1 \mathrm{~km}$ ) a été utilisée pour générer le relief du modèle à $2,5 \mathrm{~km}$. Pour cette raison, nous distinguons dans l'analyse des résultats les hivers 2010/2011 et 2011/2012 d'une part, des hivers 2012/2013 et 2013/2014, d'autre part.

\section{Méthodologie}

Afin de réaliser une évaluation objective du modèle Arome en zone de montagne, les prévisions des paramètres météorologiques, tels que la température à $2 \mathrm{~m}$ ou les précipitations hivernales, ont été comparées aux mesures issues des stations automatiques d'altitude (stations Nivoses et Radome), et des postes nivométéorologiques. Seules les stations dont l'écart entre l'altitude réelle et celle du modèle est inférieur à $150 \mathrm{~m}$ ont été retenues. La localisation de ces points de mesures est présentée sur les figures $2 \mathrm{a}$ et $2 \mathrm{~b}$ pour les Alpes et les Pyrénées. Ces paramètres ont également été comparés à ceux issus des réanalyses Safran (Durand et al., 2009, voir encadré 1). Ces réanalyses ont été interpolées sur la grille à $0,025^{\circ}$ selon la méthode présentée dans Vionnet et al. (2012). Des scores statistiques tels que le biais et l'écarttype (ECT) ont été calculés. En outre, les scores de compétence (voir encadré 2) utilisés quotidiennement pour évaluer les précipitations prévues sur la France ont été calculés sur les massifs alpins et pyrénéens.

\section{Température}

Les biais et les écarts-types entre les prévisions Arome et les observations ont été calculés par tranche d'altitude (huit stations en dessous de $1500 \mathrm{~m}, 17$ stations entre 1500 et $2500 \mathrm{~m}$ et huit stations au-dessus de $2500 \mathrm{~m}$ ). Le cycle diurne de ces scores pour les stations alpines est représenté sur la figure 3. En dessous de $1500 \mathrm{~m}$ d'altitude, les prévisions de températures d'Arome présentent un biais négatif de moins de $1{ }^{\circ} \mathrm{C}$ par rapport aux observations, peu variable selon le moment de la journée pour les deux périodes de l'étude. En revanche, entre 1500 et $2500 \mathrm{~m}$, le

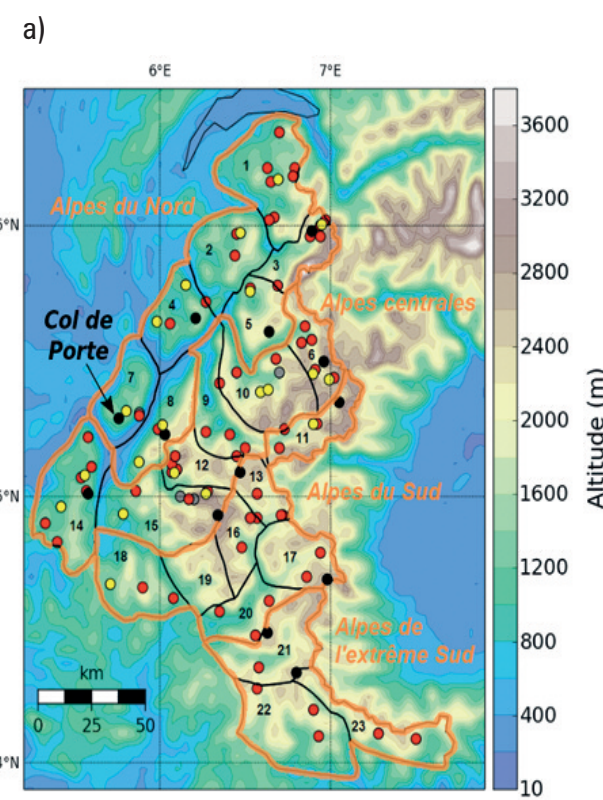

Figure 2. a) Les Alpes françaises. Localisation des stations de mesure au sol de température (en jaune), température et hauteur de neige (en noir), précipitation et hauteur de neige (en rouge), précipitation (en gris). Les limites des massifs Safran sont tracées en noir. b) Les Pyrénées. Localisation des stations de mesure de précipitation et hauteur de neige (en rouge), de hauteur de neige (en noir) et d'équivalent en eau (en bleu). Les limites des massifs Safran sont précisées.

$\begin{array}{lll}\text { Alpes du Nord } & \text { Alpes centrales } & \text { Alpes du Sud } \\ \text { 1. Chablais } & \text { 6. Haute-Tarentaise } & \text { 11. Haute-Maurienne } \\ \text { 2. Aravis } & \text { 9. Maurienne } & \text { 13. Thabor } \\ \text { 3. Mont-Blanc } & \text { 10. Vanoise } & \text { 14. Vercors } \\ \text { 4. Bauges } & \text { 12. Grandes-Rousses } & \text { 16. Pelvoux } \\ \text { 5. Beaufortain } & \text { 15. Oisans } & \text { 17. Queyras } \\ \text { 7. Chartreuse } & & \text { 18. Devoluy } \\ \text { 8. Belledonne } & & \text { 19. Champsaur } \\ \text { b) } & & \text { 20. Parpaillon }\end{array}$

Alpes de l'extrême sud

21. Ubaye 22. Alpes azuréennes 23. Mercantour

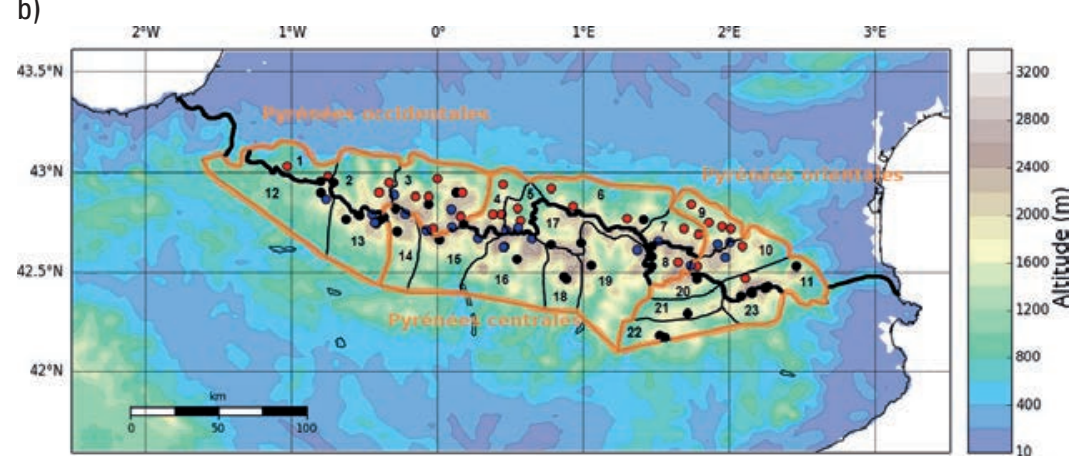

Pyrénées occidentales

1. Pays Basque

2. Aspe-Ossau

3. Bigorre

12. Navarra

13. Jacetania
Pyrénées centrales 4. Aure-Louron

5. Luchonnais

6. Couserans

7. Haute-Ariège

8. Andorre

14. Gallego

15. Sobrarbe

16. Esera

17. Aran

18. Ribagorca

19. Pallaresa
Pyrénées orientales

9. Orlu St-Barthélémy

10. Capcir-Puymorens

11. Cerdagne

20. Perafita

21. Cadi-Moixero

22. Prepirene

23. Ter-Frese 


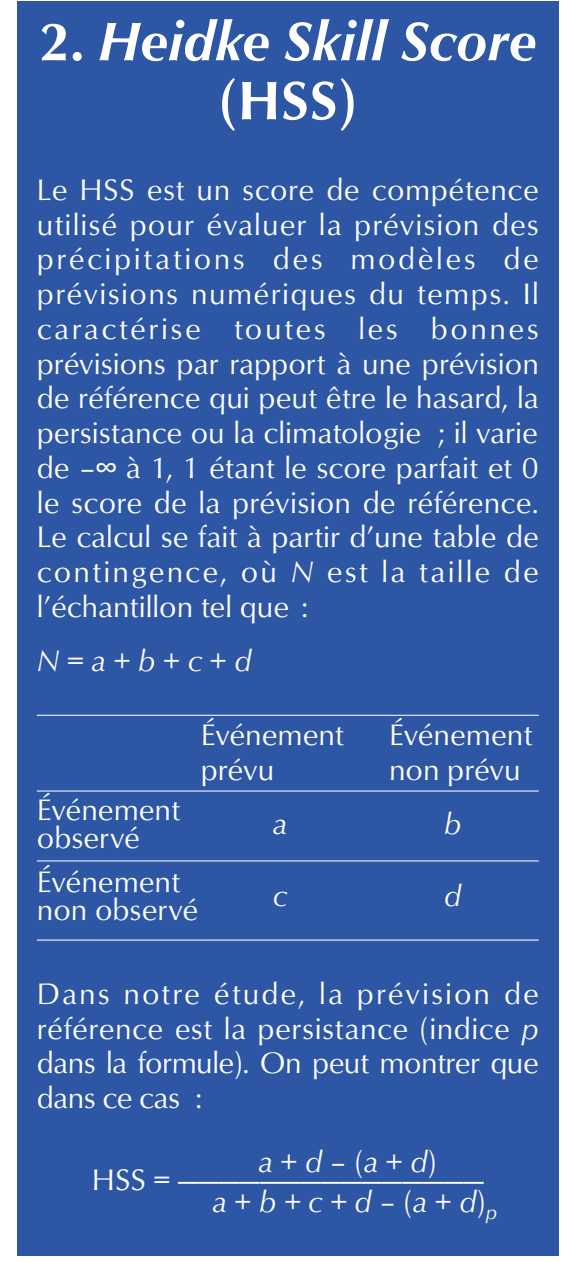

biais présente un cycle diurne marqué et peut atteindre $-3{ }^{\circ} \mathrm{C}$ peu avant l'aube. Ce biais est plus fortement négatif audelà de $2500 \mathrm{~m}$ d'altitude. Toutefois, le biais en température pour les altitudes élevées diminue légèrement pour la période 2012-2014 par rapport à la période 2010-2012. Ceci peut s'expliquer par les nombreuses améliorations du modèle Arome au cours des premières années d'utilisation opérationnelle.

Pour les altitudes élevées (supérieures à $2500 \mathrm{~m}$ ), les biais ont été également calculés pour quatre mois de l'année (janvier, avril, août et novembre). Ils sont représentés sur la figure 4. Le biais froid est plus important en hiver qu'en été, suggérant une influence de la couverture neigeuse. Il faut cependant noter que le cycle diurne est le même toute l'année. Trois causes possibles à ce biais négatif ont été identifiées et discutées en détails dans l'étude de Vionnet et al. (2016). La première de ces causes est une sousestimation du rayonnement infrarouge incident liée à une sous-estimation de la nébulosité par le modèle. Ce comportement a été mis en évidence au niveau de deux stations d'altitude et une étude plus détaillée est en cours afin de mieux quantifier les erreurs de

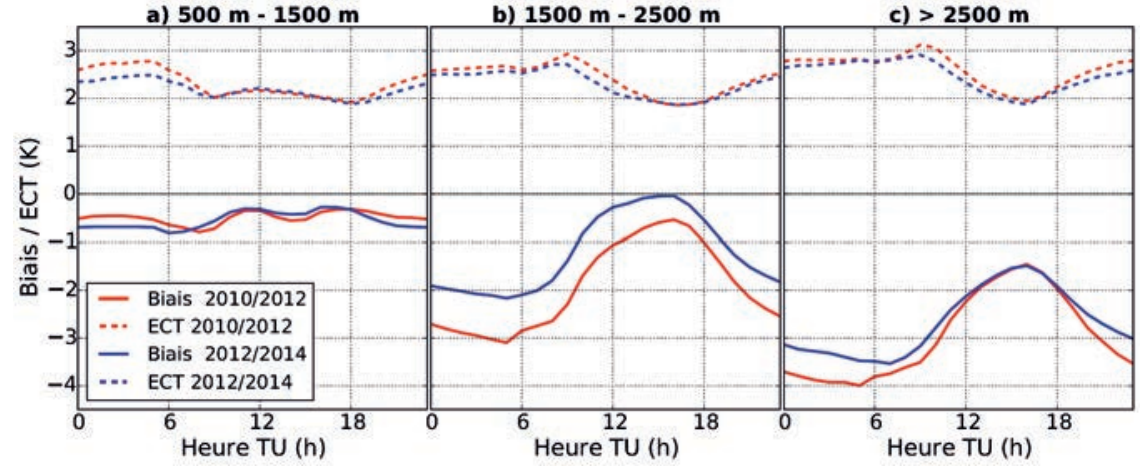

Figure 3. Biais et écart-type de l'erreur (en K) pour trois tranches d'altitude pour les prévisions Arome de la température à $2 \mathrm{~m}$ dans les Alpes. Deux périodes sont considérées : 2010-2012 (en rouge) et 2012-2014 (en bleu).

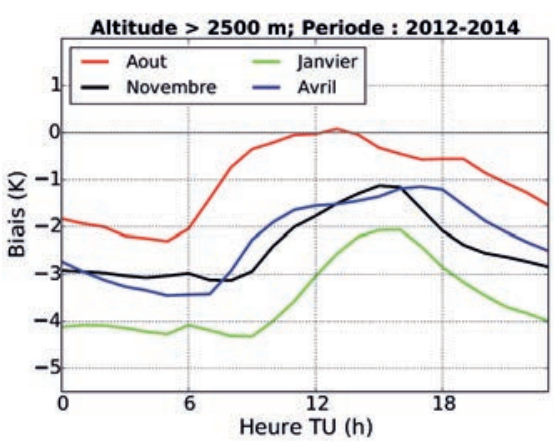

Figure 4. Cycle diurne du biais de température (en K) pour la tranche d'altitude supérieure à $2500 \mathrm{~m}$ pour quatre mois de la période 2012 2014 : janvier, avril, août et novembre.

rayonnement d'Arome en zone de montagne. Des améliorations sont attendues avec la nouvelle version d'Arome à 1,3 km qui prend en compte les effets d'orientation des pentes sur le rayonnement solaire incident, ce qui peut localement modifier le bilan d'énergie de surface avec des conséquences pour le diagnostic de température à $2 \mathrm{~m}$. La seconde raison du biais froid concerne le schéma de manteau neigeux à une couche (Douville et al., 1995) utilisé dans Arome avec une sous-estimation potentielle de la conductivité thermique du manteau neigeux liée à une sousestimation de la densité de la neige à haute altitude favorisant des températures froides à la surface du manteau neigeux. Enfin, le biais froid peut être associé aux difficultés du schéma de turbulence d'Arome en conditions atmosphériques stables qui peut favoriser un découplage entre la surface et l'atmosphère (Rontu $e t$ al., 2016).

\section{Précipitations hivernales}

Pour évaluer les précipitations prévues (pluie et neige confondues) par Arome, nous nous sommes d'abord intéressés aux cumuls quotidiens sur 24 heures pour la période hivernale, restreinte du ler décembre au 31 mars afin que les précipitations soient majoritairement neigeuses : 75,5\% des précipitations prévues par Arome à la localisation des stations d'évaluation sont sous la phase neige pendant cette période. Les observations utilisées sont issues du réseau climatologique d'état, du réseau nivométéorologique et de stations automatiques de Météo-France mesurant la précipitation totale (pluie et neige) à l'aide de pluviomètres à lecture directe ou à augets basculants suivant les réseaux de mesure. Nous avons calculé le Heidke Skill Score (HSS) pour différents seuils de précipitations. C'est un score dit de compétence utilisé par la plupart des services météorologiques pour évaluer les modèles opérationnels de prévision numérique du temps (voir encadré 2). Les valeurs de ce score calculé à partir des prévisions Arome et des réanalyses Safran pour les différents seuils sur les Alpes sont représentées sur la figure 5 .

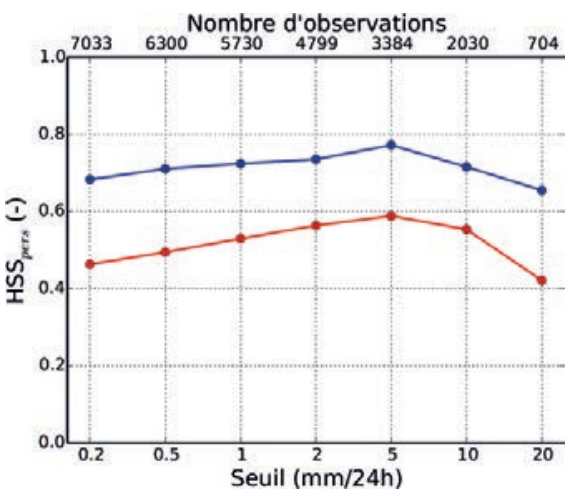

Figure 5. Heidke Skill Score (HSSpers) calculé par rapport à la persistance pour les précipitations quotidiennes prévues par Arome (rouge) et analysées par Safran (bleu) sur les Alpes pour les hivers 2010-2011 à 20132014. 
Les valeurs de HSS obtenues avec Arome pour les Alpes sont inférieures à celles obtenues avec les réanalyses Safran, ce qui s'explique par le fait que les réanalyses assimilent les observations. Nous avons fait le même constat pour les Pyrénées (Quéno et al., 2016). Toutefois, les HSS sont un peu meilleurs sur les Alpes que sur les Pyrénées. Après analyse des tableaux de contingence, les valeurs de HSS d'Arome s'expliqueraient par une surestimation de la quantité de précipitations en montagne comparées aux mesures des pluviomètres.

Les précipitations hivernales ont ensuite été évaluées en termes de cumul saisonnier de chutes de neige. En effet, l'intérêt était de savoir si Arome était capable de fournir des chutes de neige plus variables dans l'espace (variabilité intramassif) que Safran. Les cumuls de chutes de neige simulés par Arome, ceux réanalysés par Safran et la différence entre les deux cumuls sont représentés sur la figure 6 pour la saison 2012-2013, année bien enneigée. Tout d'abord, il n'y a pas de discontinuité entre les massifs sur les quantités cumulées prévues par Arome, au contraire de Safran : c'est le cas notamment entre Grandes-Rousses et Thabor et entre Ubaye et Mercantour. En moyenne, les cumuls de neige prévus par Arome sont supérieurs à ceux analysés par Safran. Toutefois, sur les versants exposés à l'est, les cumuls Arome sont en général inférieurs à ceux de Safran, comme par exemple dans les massifs du Vercors, du Thabor ou du Parpaillon. On retrouve ces différences sur les cumuls de neige pour la même année 2012-2013 sur la
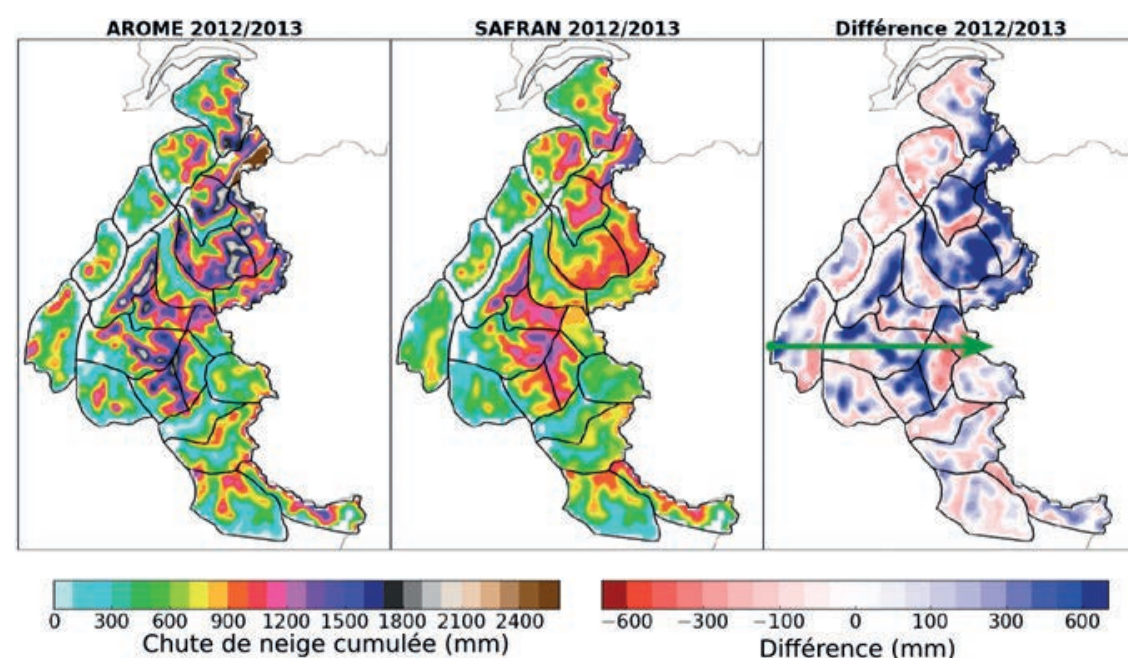

Figure 6. Chutes de neige cumulées ( $\mathrm{mm}$ d'équivalent en eau) prévues par Arome (gauche) et analysées par Safran (milieu) du $1^{\text {er }}$ novembre 2012 au 31 mai 2013. La carte de droite montre la différence Arome-Safran. La flèche verte indique la position de la coupe présentée en figure 7.

coupe topographique de la figure 7. Avec Arome, les cumuls de neige sont plus importants au vent que sous le vent des massifs du Vercors et de l'Oisans, contrairement à Safran. En effet, une des hypothèses de Safran étant l'homogénéité climatologique des massifs, il ne peut reproduire de variabilité intramassif des précipitations pour une altitude donnée. Dans les Pyrénées, on retrouve cette sensibilité des cumuls de neige prévus par Arome au flux et à l'orographie, sur la coupe topographique nord-ouest sud-est représentée sur la figure 8 , pour le 22 février 2012. Les cumuls de neige Arome sont plus importants sur le massif de la Haute-Bigorre que sur le massif espagnol de Esera. En revanche, les cumuls de neige analysés par Safran sont équivalents entre les deux versants français et espagnol.

\section{Synthèse intermédiaire}

Les résultats de cette première évaluation des paramètres météorologiques simulés par Arome en zone de montagne montrent qu'il existe un biais négatif sur la température à $2 \mathrm{~m}$ en altitude. En outre, les scores de réussite sur les précipitations sont moins bons que pour la réanalyse Safran. En revanche, contrairement à Safran, la dynamique d'Arome lui permet de reproduire des impacts du relief sur les précipitations, notamment neigeuses (effet de foehn, continuité intermassif), qui manquent à Safran. Des simulations du manteau neigeux alimentées par Arome ont donc été réalisées sur les quatre années nivologiques et sur les Alpes françaises et les Pyrénées, en ayant à l'esprit les conclusions de cette étude.

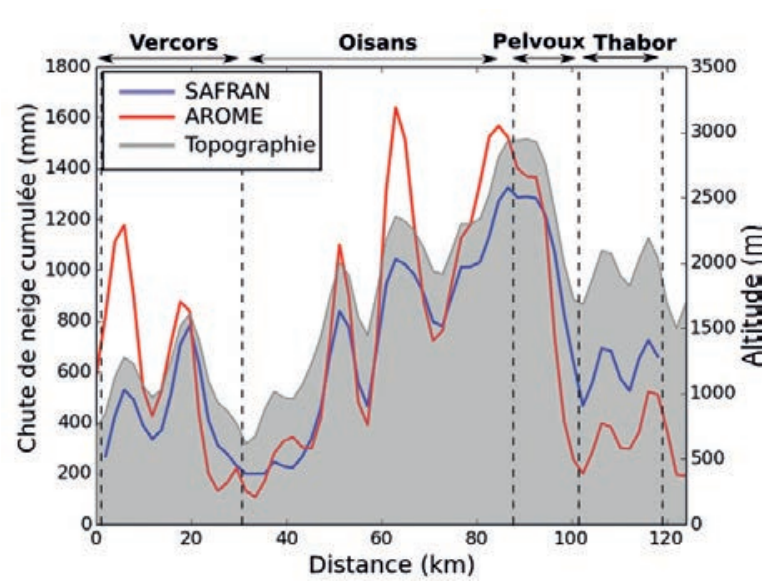

Figure 7. Coupe ouest-est montrant les chutes de neige cumulées (mm d'équivalent en eau) prévues par Arome (rouge) et analysées par Safran (bleu). La localisation de la coupe topographique est précisée sur la figure 6. La zone grise représente le relief (échelle de droite).

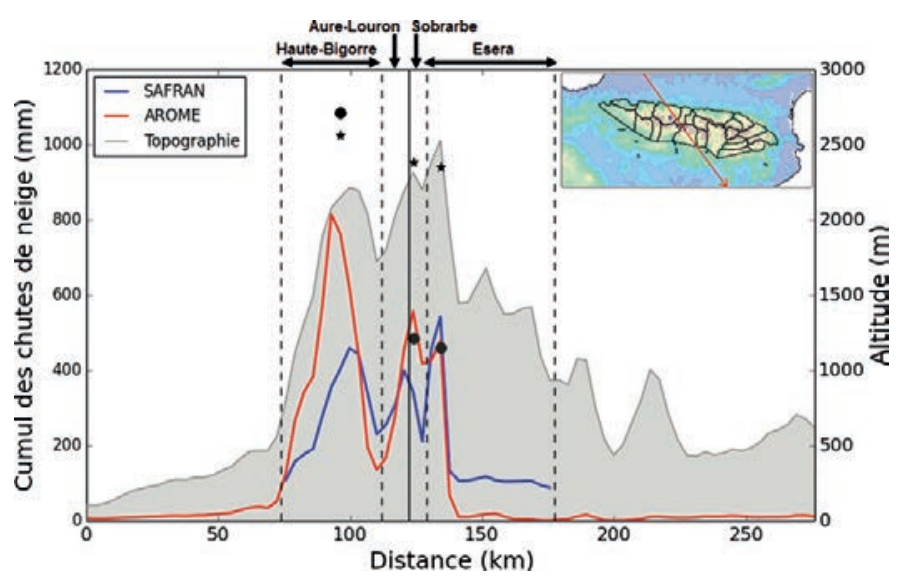

Figure 8. Coupe nord-est sud-ouest à travers les Pyrénées (encart en haut à droite) montrant les chutes de neige cumulées (mm d'équivalent en eau) du $1^{\text {er }}$ novembre 2011 au 22 février 2012 simulées par Arome (rouge), analysées par Safran (bleu) et mesurées à trois stations situées le long du transect (ronds noirs). Les étoiles indiquent l'altitude réelle de chaque station. La zone grise représente le relief (échelle de droite). 


\section{Vers une chaîne Arome/Surfex-Crocus}

Les prévisions 24 heures d'Arome pour les quatre années nivologiques évaluées précédemment ont été combinées afin de fournir des forçages atmosphériques continus à Surfex- Crocus. Les résultats de ces simulations ont été évalués en termes de hauteurs de neige et d'équivalents en eau de la neige (EEN) sur les Alpes et les Pyrénées, via des calculs de scores : biais, écart-type, mais aussi variation journalière de la hauteur de neige (notée $\triangle \mathrm{HTN}=$ hauteur de neige du jour $J$ - hauteur de neige du jour $J-1)$. Les mesures ponctuelles de hauteur de neige sont issues des postes du réseau nivométéorologique où une mesure quotidienne de hauteur de neige est réalisée, ainsi que des stations automatiques Nivose de haute montagne où la hauteur de neige est mesurée automatiquement à l'aide de capteur à ultrasons. La localisation des stations est mentionnée sur la figure 2 . Par ailleurs, les données d'équivalent en eau de la neige disponibles au col de Porte (figure 2a) sont obtenues à l'aide d'un NRC (nivomètre à rayonnement cosmique, capteur au sol de flux neutronique du rayonnement cosmique qui considère l'atténuation de ce dernier par l'atmosphère puis le manteau neigeux pour en déduire son équivalent en eau) développé par EDF (Gottardi et al., 2013). Enfin, les images de fractions neigeuses quotidiennes issues des données satellitaires de Terra-Modis (MOD10A1, Klein et Stroeve, 2002) à la résolution horizontale de $500 \mathrm{~m}$ ont également été utilisées afin d'évaluer spatialement les simulations de manteau neigeux.

\section{Validation au col de Porte}

Afin d'avoir une première idée du comportement d'une chaîne de modélisation Arome/Surfex-Crocus (notée Arome-SC), les hauteurs de neige et les équivalents en eau de la neige simulés ont été comparés aux mesures du col de Porte, dans le massif de la Chartreuse, au nord de Grenoble. Ce site expérimental permet, depuis les années 1960, d'acquérir les données météorologiques et nivologiques utiles notamment à l'évaluation de la modélisation du manteau neigeux. La figure 9 montre, pour les quatre saisons nivologiques, les hauteurs de neige et les équivalents en eau simulés par la chaîne Arome-

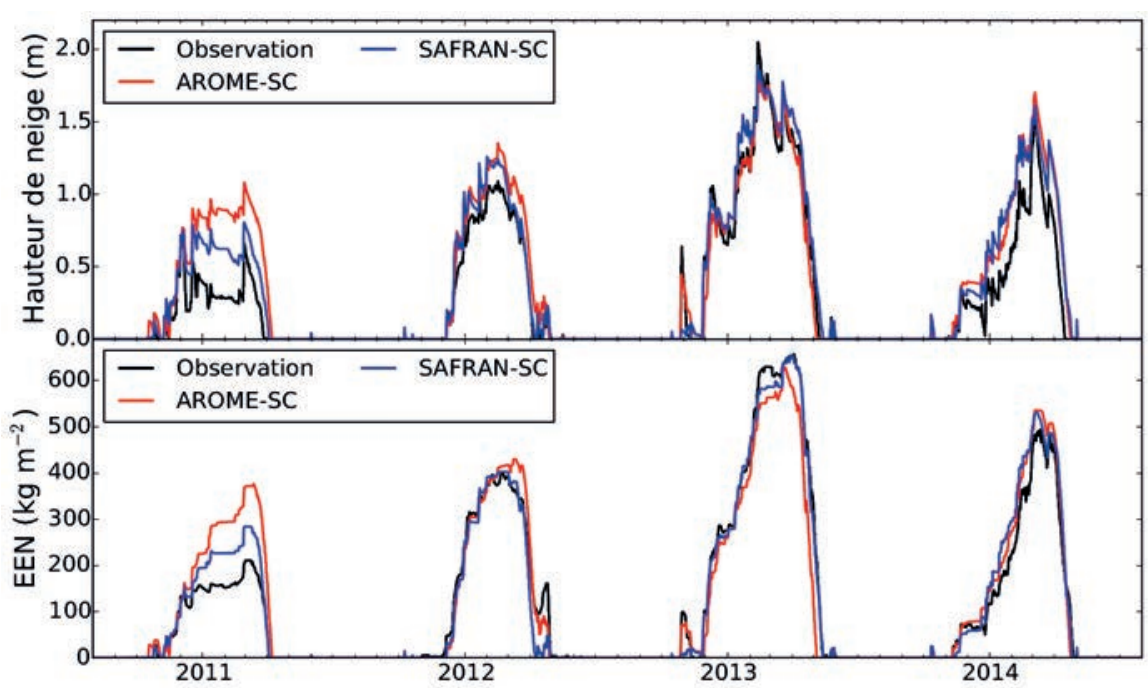

Figure 9. Évolution temporelle de la hauteur de neige et de l'équivalent en eau de la neige (EEN) observés et simulés au col de Porte par Arome-SC et Safran-SC pour les hivers 2010-2011 à 20132014.

SC, comparés aux observations et aux résultats de la chaîne Safran/SurfexCrocus (notée Safran-SC et utilisant en entrée de Crocus les réanalyses Safran décrites précédemment). Les hauteurs de neige ne sont pas correctement simulées pour la saison 2010-2011 en raison d'une erreur sur la phase des précipitations pour deux épisodes. En revanche, pour les autres hivers, Arome-SC reproduit bien la succession de périodes d'accumulation et de fonte. En outre, les écarts sont du même ordre que ceux obtenus avec Safran-SC. Ces résultats très encourageants doivent être regardés sur l'ensemble des Alpes et des Pyrénées.

\section{Hauteur de neige}

Les biais et les écarts-types ont été calculés sur les régions alpines françaises et pyrénéennes (total de 162 stations) et sont reportés dans le tableau 1. On constate qu'Arome-SC a tendance à surestimer les hauteurs de neige sur les Alpes françaises et les Pyrénées. Néanmoins, comme pour les précipitations hivernales, les biais sont plus importants dans les Pyrénées que dans les Alpes. De plus, on observe une décroissance nord-sud du biais dans les Alpes (en phase avec la diminution des quantités de précipitations, figure 5). Dans les Pyrénées, cette décroissance est orientée ouest-est, et est là aussi en phase avec le régime des précipitations.

\section{Précipitations et hauteurs de neige : un paradoxe ?}

Comme on l'a montré précédemment, Arome-SC semble surestimer les hauteurs de neige. Afin d'étudier quotidiennement le comportement de Arome-SC, les variations journalières de hauteurs de neige (notées $\Delta \mathrm{HTN}$ ) ont été calculées sur les quatre années sur

Tableau 1. Scores sur les hauteurs de neige (biais et écart-type en $\mathrm{cm}$ ) pour les quatre saisons hivernales.

\begin{tabular}{lccccc} 
Région & Nombre & \multicolumn{2}{c}{ Arome-SC } & \multicolumn{2}{c}{ Safran-SC } \\
\cline { 3 - 6 } de stations & Biais & Écart-type & Biais & Écart-type \\
Alpes du Nord & 23 & 51 & 46 & 23 & 38 \\
\hline Alpes centrales & 30 & 50 & 49 & 21 & 36 \\
\hline Alpes du Sud & 19 & 25 & 51 & 15 & 37 \\
\hline Alpes extrême Sud & 7 & 8 & 31 & -1 & 29 \\
\hline Alpes françaises & $\mathbf{7 9}$ & $\mathbf{4 0}$ & $\mathbf{5 0}$ & $\mathbf{1 8}$ & $\mathbf{3 7}$ \\
\hline Pyrénées occidentales & 27 & 65 & 84 & 17 & 54 \\
\hline Pyrénées centrales & 35 & 57 & 64 & 28 & 55 \\
\hline Pyrénées orientales & 21 & 36 & 58 & 18 & 63 \\
\hline Pyrénées & $\mathbf{8 3}$ & $\mathbf{5 5}$ & $\mathbf{7 0}$ & $\mathbf{2 2}$ & $\mathbf{5 7}$ \\
\hline
\end{tabular}


les Pyrénées. Sur la figure 10, elles sont représentées, classées par catégories, et comparées à celles de Safran-SC et aux observations. Les valeurs négatives correspondent à une diminution quotidienne de la hauteur de neige par tassement et/ou ablation du manteau neigeux (fonte ou érosion par le vent ou sublimation). Safran-SC et Arome-SC sous-estiment le nombre de fortes décroissances $(>10 \mathrm{~cm}$ ) par rapport aux observations. Une partie se retrouve dans les faibles décroissances, tandis que l'autre peut s'expliquer par l'absence de simulation du transport de neige par le vent dans la version opérationnelle de Crocus et la sousestimation des fortes fontes. Pour ce qui est des accumulations, Safran$\mathrm{SC}$ est en deçà des observations à partir d'accumulations supérieures à $10 \mathrm{~cm}$ et l'écart augmente avec $\Delta$ HTN. $A$ contrario, le nombre de $\Delta \mathrm{HTN}$ simulées par Arome-SC reste proche du nombre d'observations. Par conséquent, Arome- SC surestimerait les hauteurs de neige par manque d'ablation, tandis que pour les accumulations il serait proche des observations, à l'exception des accumulations supérieures à $100 \mathrm{~cm}$.

Or, notre évaluation des précipitations hivernales prévues par Arome concluait à une surestimation de ces précipitations par Arome. D'ailleurs, si on examine la figure 11 qui représente la fréquence des précipitations quotidiennes par catégorie, on arrive à la même conclusion. Ce paradoxe entre surestimation des précipitations et sous-estimation de l'accumulation de neige a été mis en évidence par Schirmer et Jamieson (2015) avec deux modèles équivalents à Arome et Crocus, respectivement le modèle météorologique canadien GEM-LAM et le modèle de manteau neigeux Snowpack. En outre, ces résultats sont identiques avec l'équivalent en eau à la place de la hauteur de neige (non présentés ici). En fait, ceci s'explique par la sous-captation de la mesure des précipitations solides par les pluviomètres lors de vent modéré à fort. L'erreur peut être comprise entre 30 à $70 \%$, selon la température, la orce du vent, etc. Une correction peut être appliquée, mais l'erreur n'est pas totalement corrigée (Wolff et al., 2015 ; WMO-SPICE, 2015). En conclusion, les hauteurs de neige et les mesures d'équivalents en eau peuvent apporter une information complémentaire aux mesures des pluviomètres pour l'analyse (au sens assimilation de données) des précipitations en montagne.

\section{Variabilité de la couverture neigeuse}

Afin d'évaluer la capacité d'Arome-SC à représenter la variabilité de la couverture neigeuse (c'est-à-dire la présence ou non de neige au sol), celleci a été comparée a été comparée à la fraction neigeuse issue de Modis projetée sur la grille Arome. Les cartes de fraction neigeuse issues de Modis et les cartes de couverture neigeuse simulée par Arome-SC et Safran-SC ont été tout d'abord visualisées. C'est le cas notamment pour le 22 février 2012, sur la figure 12. Alors que Safran-SC simule un enneigement plutôt homogène sur l'ensemble des Pyrénées, Arome-SC simule la quasiabsence de neige côté espagnol en accord avec Modis. L'enneigement pyrénéen de cet hiver a été essentiellement constitué par des flux nord-nord-ouest produisant de la neige côté français (au vent) et peu de neige côté espagnol (sous le vent). Arome-SC reproduit bien l'impact de l'orographie sur l'enneigement, en accord avec la coupe topographique des chutes de neige cumulées pour la même date en figure 8 .

Afin d'avoir une évaluation quantitative de la capacité d'Arome$\mathrm{SC}$ à bien reproduire la couverture neigeuse, on a utilisé l'indice de Jaccard (encadré 3). L'indice de Jaccard (noté $J$ ) est une comparaison pixel par pixel et traduit une analogie de surface $(1=$ score parfait, $0=$ pas d'analogie). Sur la figure 13 est représentée l'évolution temporelle de $J$ pour Arome-SC et Safran-SC au cours de l'hiver 2011-2012. On constate que, jusqu'au printemps (soit la période de fonte du manteau neigeux), Arome-SC a une meilleure adéquation à la fraction neigeuse de Modis que Safran-SC, puisque $\mathrm{J}$ est plus élevé. Les calculs de $J$ sur d'autres saisons hivernales et

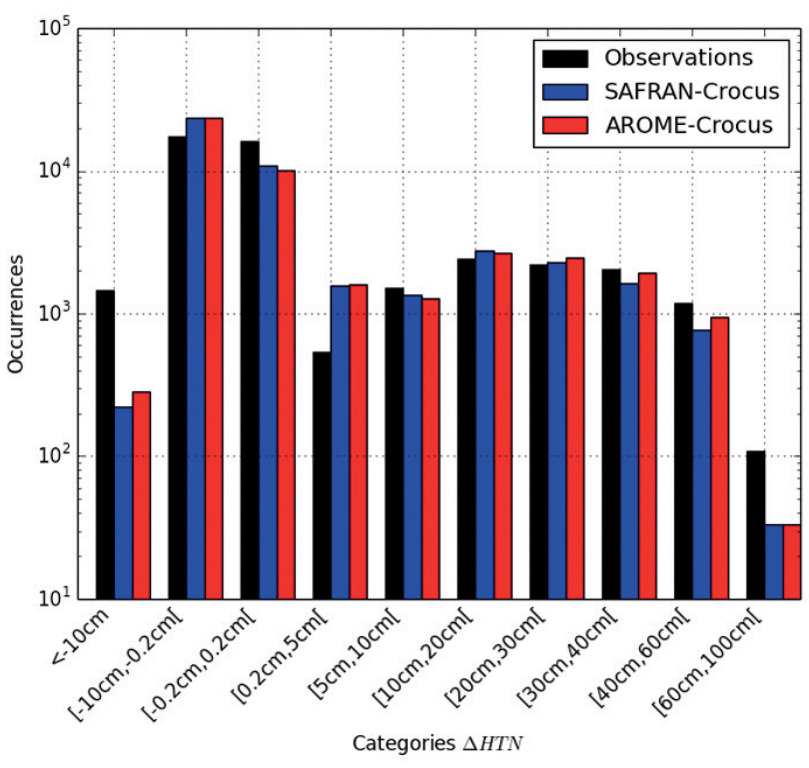

Figure 10. Distribution catégorielle des variations journalières de hauteurs de neige (notées $\triangle H T N$ ) pour les observations (en noir), Arome-SC (en rouge), Safran-SC (en bleu), sur les Pyrénées pour les quatre hivers.

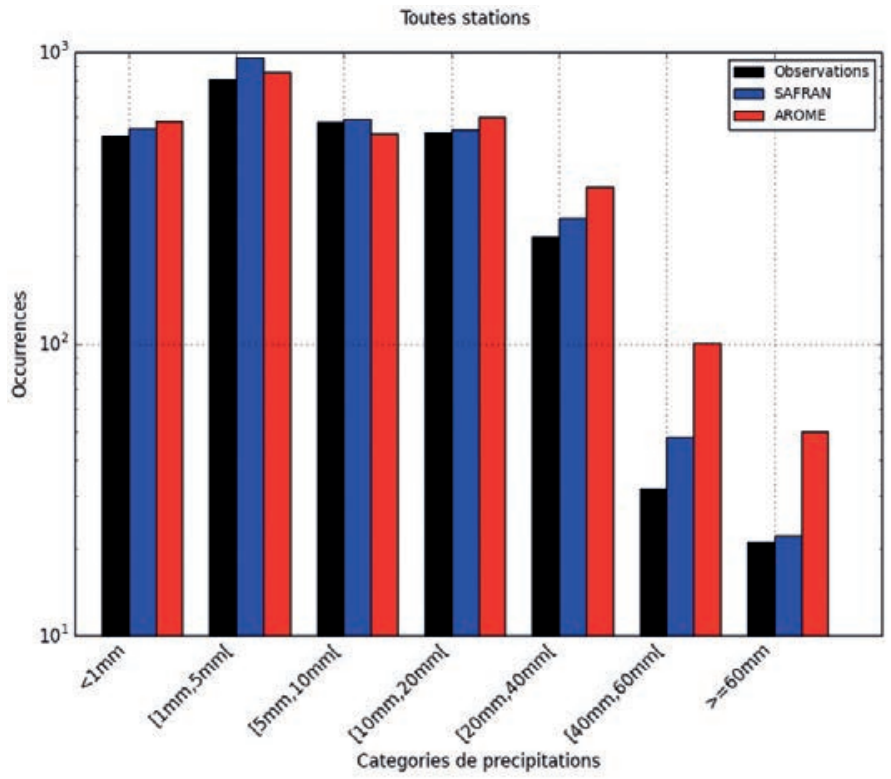

Figure 11. Distribution catégorielle des précipitations pour les observations (en noir), Arome-SC (en rouge), Safran-SC (en bleu), sur les Pyrénées pour les quatre hivers. 

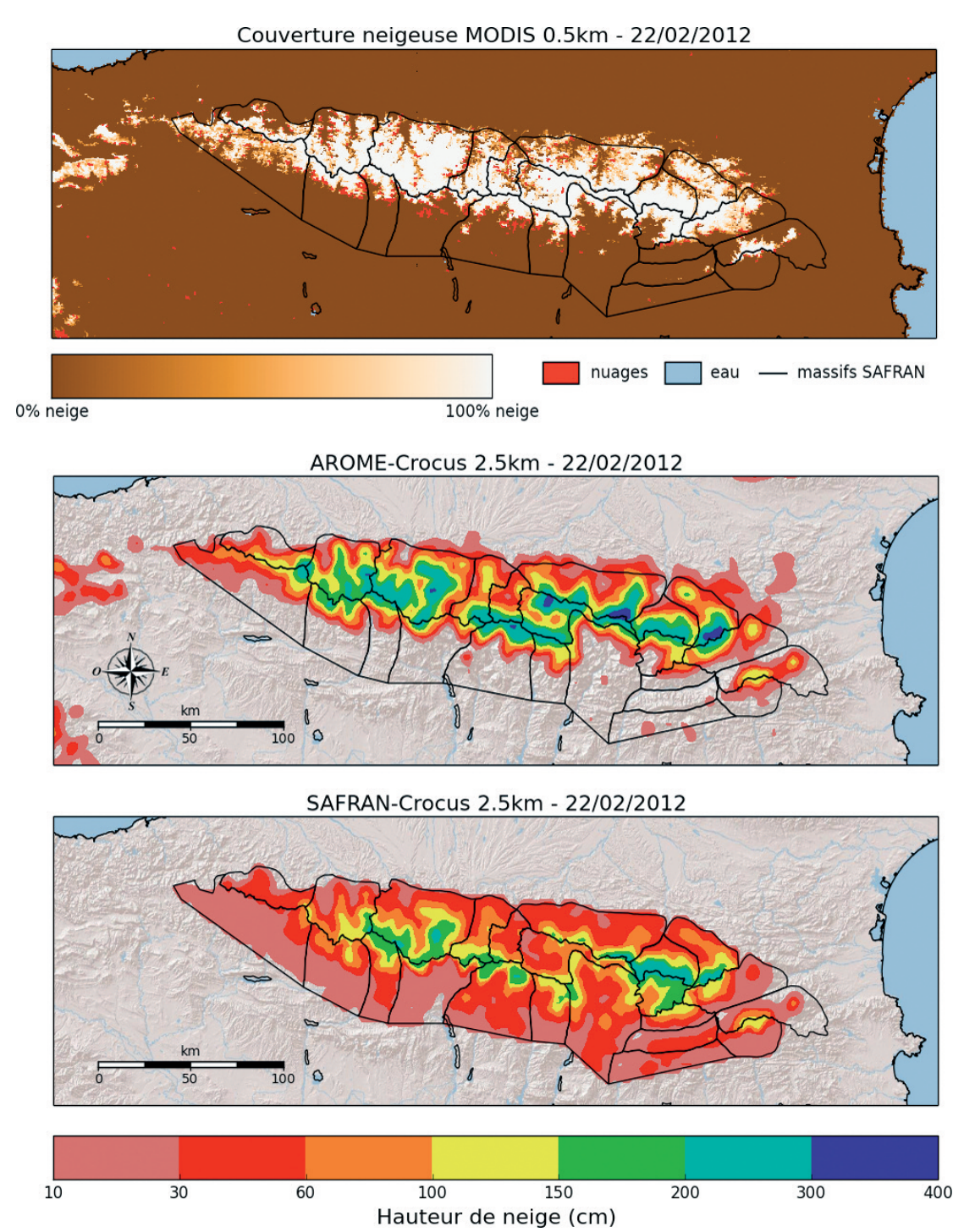

Figure 12. Couverture neigeuse issue de Modis (en haut), hauteur de neige $(\mathrm{cm})$ simulée par AromeSC (au milieu) et par Safran-SC (en bas) à la date du 22 février 2012.

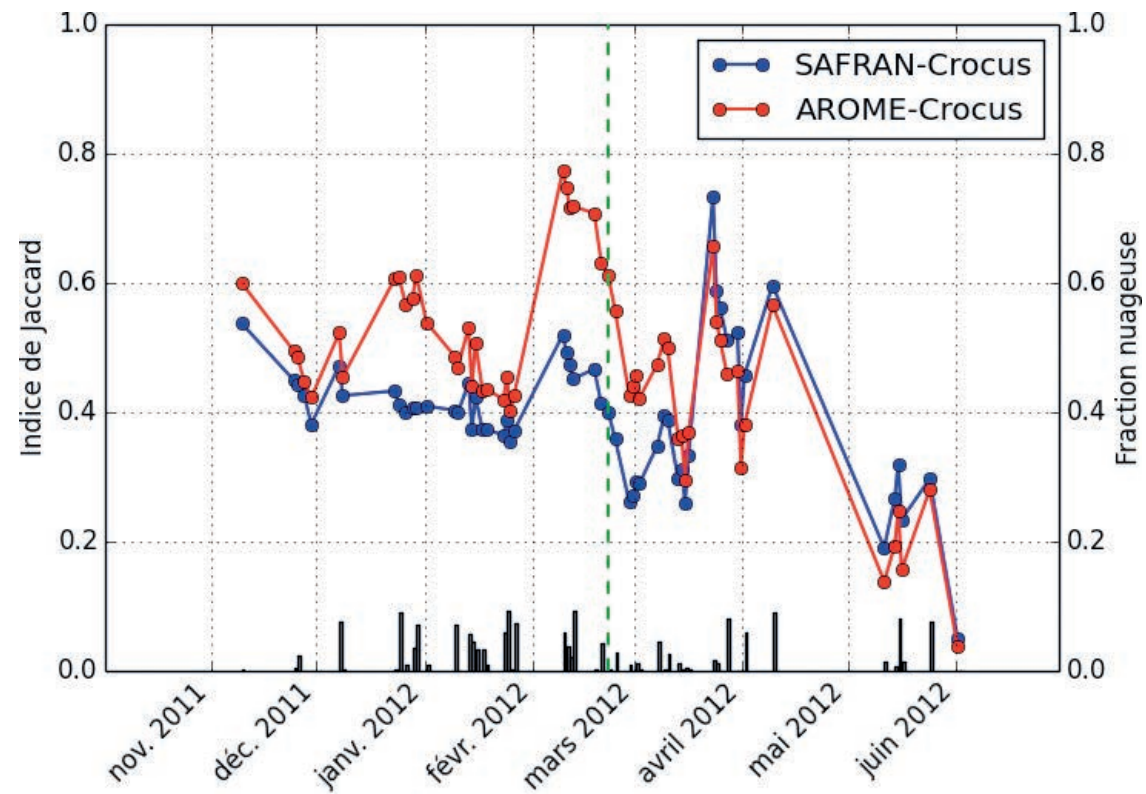

Figure 13. Évolution de l'indice de Jaccard de la couverture de neige au sol au cours de l'hiver 20112012 pour Arome-SC en rouge et Safran-SC en bleu. La fraction nuageuse est représentée par les barres noires. La ligne tiretée verte correspond à la date du 22 février 2012, représentée en figure 10.

\section{Indice de Jaccard $(J)$}

J correspond au nombre de pixels communs aux surfaces A (domaine de

la couverture de neige simulée) et B (domaine de la couverture neigeuse observée), divisé par le nombre de pixels dans I'union des deux surfaces :

\section{$J=\frac{|A \cap B|}{|A \cup B|}$ \\ $\overline{|A \cup B|}$}

$J$ est compris entre 0 et $1: 0$ signifie qu'il n'y a pas de chevauchement des surfaces $A$ et $B ; 1$ signifie que $A=B$.

d'autres calculs de scores sur la répartition de l'enneigement aboutissent à la même conclusion. Ainsi, l'utilisation d'Arome améliore la répartition spatiale de l'enneigement simulé.

\section{Conclusions et perspectives}

L'objectif de ces travaux de recherche était d'évaluer le comportement du modèle de prévision météorologique à résolution kilométrique, Arome, en zone de montagne. Il fallait d'abord déterminer si les prévisions météorologiques fournissaient des paramètres météorologiques (température, humidité, rayonnement, vent, précipitations) en accord avec les observations. Nous avons pu constater que les prévisions Arome présentent un biais froid en altitude. Au niveau des précipitations hivernales, Arome a tendance à les surestimer en altitude par rapport aux observations conventionnelles (pluviomètres), mais il faut modérer ceci par la sousestimation de la mesure des précipitations solides. Pour les paramètres météorologiques non présentés dans cet article, mais également évalués, on peut noter que la vitesse du vent prévu est non biaisée et que le cycle diurne de l'humidité relative est bien représenté. En revanche, le rayonnement solaire est surestimé et le rayonnement infrarouge sous-estimé, en raison notamment d'une sous-estimation de la couverture nuageuse. Ceci explique en partie le biais froid d'altitude. Une discussion plus approfondie sur cette évaluation météorologique a été publiée dans Vionnet et al. (2016). 
Les simulations du manteau neigeux par Surfex-Crocus forcé par les prévisions Arome ont été comparées aux simulations effectuées avec les réanalyses Safran et aux observations in situ. Sur le site de référence du col de Porte, les hauteurs de neige (et l'équivalent en eau de la neige) issues d'Arome-SC sont comparables à celles de Safran-SC et proches des observations. En revanche, sur l'ensemble des Alpes et des Pyrénées, les scores sont plus contrastés. Le biais sur les hauteurs de neige est de $40 \mathrm{~cm}$ sur les Alpes (contre $18 \mathrm{~cm}$ pour Safran-SC) et de $55 \mathrm{~cm}$ sur les Pyrénées (contre $22 \mathrm{~cm}$ pour SafranSC). Ces erreurs s'expliquent par la sous-estimation de la fonte et l'absence de transport de neige par le vent dans Surfex-Crocus. En outre, SafranSC ayant tendance à être en deçà des observations lors de fortes accumulations, contrairement à Arome$\mathrm{SC}$, les deux erreurs se compensent et les scores sont meilleurs que pour Arome-SC.

L'intérêt de cette étude est aussi de savoir si les particularités d'Arome lui permettaient, associé à Surfex-Crocus, de mieux reproduire la variabilité spatiale de l'enneigement que SafranSC. Pour cela, les enneigements simulés par Arome-SC et Safran-SC ont été comparés à la couverture neigeuse issue de l'instrument Modis (à bord de Terra) à l'aide d'indices de similarité de surface, tel que l'indice de Jaccard. Arome-SC représente mieux la répartition spatiale de l'enneigement que Safran-SC au cours de la saison nivologique, hormis au moment de la fonte où les deux modèles ont des valeurs d'indices très proches. La capacité d'Arome à mieux représenter les effets du relief, notamment la répartition des précipitations entre le côté au vent et celui sous le vent, est donc mise en évidence via l'enneigement saisonnier. Les travaux sur les simulations de l'enneigement dans les Pyrénées sont détaillés par Quéno et al. (2016).

Arome est sans cesse en développement afin d'améliorer les prévisions météorologiques. Deux modifications auront un impact en zone montagneuse : d'une part, depuis avril 2015, Arome est passé de 2,5 km à $1,3 \mathrm{~km}$ de résolution horizontale avec une nouvelle modification de la base de données de relief (Brousseau et al., 2016). D'autre part, les effets des ombres portées sur le rayonnement solaire descendant sont désormais pris en compte dans le modèle. Par la suite, il faudra disposer d'une analyse météorologique adaptée en zone de montagne, à l'échelle kilométrique et basée sur Arome afin d'améliorer le forçage météorologique fourni à Crocus. Parmi les recherches en cours sur ce sujet, on peut citer l'utilisation de l'information issue des nouveaux radars bande $\mathrm{X}$ installés dans les Alpes par le système Odyc (outil 1D-Var d'analyse des précipitations en zones de montagnes, Birman et al., 2017) ou encore la modification du code d'analyse pour la surface d'Arome, afin de l'adapter au relief (système Mescan, Soci et al., 2016). Des travaux ont débuté sur une assimilation pertinente des données satellitaires de rayonnement issues de LandSAF. Il faudra également prendre en compte les hauteurs de neige et les équivalents en eau qui ont montré leur intérêt pour l'analyse des précipitations. In fine, l'utilisation d'une analyse météorologique quotidienne basée sur l'ébauche Arome permettra de s'affranchir des "erreurs » de prévisions dans les simulations distribuées du manteau neigeux.

\section{Remerciements}

Ce travail a été réalisé grâce à de nombreuses collaborations. Les auteurs remercient M. Amodei, E. Bazile, C. Coléou, A. Colomb, M. Dumont, F. Karbou, M. Lafaysse, A. Mary, S. Morin, L. Pfitzner, I. Sanchez, Y. Seity et B. Vincendon (CNRM, MétéoFrance / CNRS, Toulouse et Grenoble) pour l'aide apportée, les conseils donnés et les discussions animées. Ils remercient également D. Six (LGGE, Grenoble), F. Gottardi (EDF/ DTG, Grenoble), S. Gascoin (Cesbio, Toulouse), J. Revuelto (Instituto Pirenaico de Ecología, Espagne) et R. Mott (WSL-SLF, Davos) pour les conseils et les échanges de données.

\section{Bibliographie}

Baldauf M., Seifert, A., Förstner, J., Majewski, D., Raschendorfer, M., Reinhardt, T., 2011. Operational convective-scale Numerical Weather Prediction with the COSM0 model: Description and sensitivities. Mon. Weather Rev., 139,3887-3905. doi: 10.1175/MWR-D-10-05013.1

Birman C., Karbou F., Mahfouf J.-F., Lafaysse M., Durand Y, Giraud G., Merindol L., Hermozo L., 2017. Analysis of precipitation in the French Alps using a variational approach and study of potential added value of ground based radar observations. J. Hydrometeorol., 18, 1425-1451. doi: 10.1175/JHM-D-16-0144.1

Bouttier F., 2007. Arome, avenir de la prévision régionale. La Météorologie, 58,12-20.

Brousseau P. Bouttier F., Hello G., Seity Y., Berre L., Montmerle T., Auger L., Malardel S., 2008. A prototype convective-scale data assimilation system for operation: The AROME-RUC. HIRLAM Tech. Rep. 68, 23-30.

Brousseau P., Seity Y., Ricard D., Léger J., 2016. Improvement of the forecast of convective activity from the AROME-France system. Q. J. R. Meteorol. Soc., 142, 22312243. doi: 10.1002/qj.2822

Brun E., Vionnet V., Morin S., Boone A., Martin E., Faroux S., Le Moigne P., Willemet J.-M., 2012. Le modèle de manteau neigeux Crocus et ses applications. La Météorologie, 76, 44-54.

Clark A.J., Weiss S.J., Kain J.S., Jirak I.L., Coniglio M., Melick C.J., Siewert C., Sobash R.A., Marsh P.T., Dean A.R., Xue M., Kong F., Thomas K.W., Wang Y., Brewster K., Gao J., Wang X., Du J., Novak D.R., Barthold F.E., Bodner M.J., Levit J.J., Entwistle C.B., Jensen T.L. Correia Jr. J., 2012. An overview of the 2010 Hazardous Weather Testbed Experimental Forecast Program Spring Experiment. Bull. Am. Meteorol. Soc., 93, 55-74.

Chow F.K., de Wekker S.F, Snyder B.J., 2013. Mountain weather research and forecasting: recent progress and current challenges. Springer.

Douville H., Royer J.-F., Mahfouf J.-F., 1995. A new snow parametrization for the Meteo-France climate model. Part 1: Validation in stand-alone experiments. Clim. Dyn., 12, 449-466.

Durand Y., Brun E., Mérindol L., Guyomarc'h G., Lesaffre B., Martin E., 1993. A meteorological estimation of relevant parameters for snow models. Ann. Glaciol., 18, 65-71.

Durand Y., Giraud G., Laternser M., Etchevers P., Mérindol L., Lesaffre B., 2009. Reanalysis of 47 years of climate in the French Alps (1958-2005): Climatology and trends for snow cover. J. Appl. Meteorol. Climatol., 48, 2487-2512. doi: 10.1175/2009JAMC1810.1 
Fischer C., Montmerle T., Berre L., Auger L., Stefanescu S.E., 2005. An overview of the variational assimilation in the Aladin/France NWP system. Q. J. R. Meteorol. Soc., 131, 3477-3492.

Gottardi F., Paquet E., Carrier P., Laval M.-T., Gailhard J., Garcon R., 2013. A decade of snow water equivalent monitoring in the French Mountain ranges, in: Proceedings of the International Snow Science Workshop Grenoble-Chamonix, 2013, 7-11 October, Grenoble, 926-930.

Kiktev D.B., Astakhova, E.D., Blinov, D.V., Zaripov R.B., Murav'ev A.V, Rivin G.S., Rozinkina I.A., Smirnov A.V., Tsyrul'nikov M.D., 2013. Development of forecasting technologies for meteorological support of the Sochi-2014 Winter Olympic Games. Russian. Russian Meteorol. Hydrol., 38, 653-660. doi: 10.3103/S1068373913100014

Klein A.G., Stroeve J., 2012. Development and validation of a snow albedo algorithm for the MODIS instrument, Ann. Glaciol., 34, 45-52. doi: $10.3189 / 172756402781817662$

Lafore J., Stein J., Asencio N., Bougeault P., Ducrocq V., Duron J., Fischer C., Hereil P., Mascart P., Masson V., Pinty J. P., Redelsperger J.-L., Richard E., Vila-Guerau de Arellano J., 1998. The Meso-NH atmospheric simulation system. Part l: Adiabatic formulation and control simulations. Ann. Geophys., 16, 90-109.

Mailhot J., Milbrandt J., Giguère A., McTaggart-Cowan R., Erfani A., Denis B., Glazer A., Vallée M., 2014. An experimental high-resolution forecast system during the Vancouver 2010 Winter Olympic and Paralympic Games. Pure Appl. Geophys., 171, 209-229.

Martin E., Brun E., Durand Y., 1994. Variabilité de l'enneigement et variations du climat. La Météorologie, 5, 19-27.

Quéno L., Vionnet V., Dombrowski-Etchevers I., Lafaysse M., Dumont M., Karbou F., 2016. Snowpack modelling in the Pyrenees driven by kilometric-resolution meteorological forecasts. The Cryosphere, 10, 1571-1589. doi: 10.5194/tc-10-1571-2016

Rontu L., Wastl C., Niemela S., 2016. Influence of the details of topography on weather forecast - evaluation of HARMONIE experiments in the Sochi Olympics domain over the Caucasian mountains, Front. Earth Sci., 4, 13. doi: 10.3389/feart.2016.00013

Schirmer M., Jamieson B., 2015. Verification of analysed and forecasted winter precipitation in complex terrain. The Cryosphere, 9, 587-601. doi: 10.5194/tc-9-587-2015

Seity Y., Brousseau P., Malardel S., Hello G., Bénard P., Bouttier F., Lac C., Masson V., 2011. The Arome-France convective scale operational model. Mon. Weather Rev., 139, 976-991. doi: 10.1175/2010MWR3425.1

Soci C., Bazile E., Besson F., Landelius T., 2016. High-resolution precipitation re-analysis system for climatological purposes. Tellus, 68A, 29879. doi: 10.3402/tellusa.v68.29879

Soubeyroux J.-M., Martin E., Franchistéguy L., Habets F., Noilhan J., Baillon M., Regimbeau F., Vidal J.-P., Le Moigne P., Morel S., 2008. Safran-Isba-Modcou (SIM), un outil pour le suivi hydrométéorologique opérationnel et les études. La Météorologie, 63, 40-45.

Vionnet V., Brun E., Morin S., Boone A., Faroux S., Le Moigne P., Martin E., Willemet J.-M., 2012. The detailed snowpack scheme Crocus and its implementation in Surfex 7.2. Geosci. Model. Dev., 5, 773-791.

Vionnet V., Dombrowski-Etchevers I., Lafaysse M., Quéno L., Seity Y., Bazile E., 2016. Numerical weather forecasts at kilometer scale in the French Alps: evaluation and application for snowpack modeling. J. Hydrometeorol., 17, 2591-2614. doi: 10.1175/JHM-D-15-0241.1

Wolff M.A., Isaksen K... Petersen-Øverleir A, Ødemark K., Reitan T., Brækkan R., 2015. Derivation of a new continuous adjustment function for correcting wind-induced loss of solid precipitation: results of a Norwegian field study. Hydrol. Earth Syst. Sci., 19, 951-967. doi: 10.5194/hess-19-951-2015

WMO-SPICE, 2015. The World Meteorological Organization solid precipitation intercomparison experiment and its applications, M.E. Earle, S. Morin, R.M. Rasmussen, M.A. Wolff, D. Yang, eds. 